\title{
Analyse, Simulation und optimale Steuerung eines Dengue-Fieber-Modells mit temporärer Kreuzimmunität
}

\author{
Mark Herath ${ }^{1}$, Kurt Chudej ${ }^{1,2 *}$ \\ ${ }^{1}$ Lehrstuhl für Wissenschaftliches Rechnen, Universität Bayreuth, \\ 95440 Bayreuth, Germany; *kurt.chudej@uni-bayreuth.de \\ ${ }^{2}$ Forschungszentrum Modellierung und Simulation (MODUS), Universität Bayreuth, 95440 Bayreuth, Germany
}

\begin{abstract}
Das Dengue-Fieber ist eine Virenerkrankung, welche durch den Stich von weiblichen, infizierten Moskitos der Spezies Aedes auf den Menschen übertragen wird. Diese Krankheit tritt vorwiegend in tropischen und subtropischen Gebieten auf, circa 40 \% der Weltbevölkerung ist davon betroffen. Im Zuge des Klimawandels breitet sich die Asiatische Tigermücke (Aedes albopictus) immer weiter in Europa und insbesondere im Süden Deutschlands aus. Durch die Rückkehr von Fernreisenden wird dieser Effekt zusätzlich verstärkt. Wir untersuchen ein Kompartimentmodell mit zwei Serotypen, das eine nach einer Erstinfektion temporäre Kreuzimmunität gegen alle auftretenden Serotypen berücksichtigt. Daneben werden unterschiedliche Kontrollstrategien in das Modell integriert, welche die Anzahl der Stechmücken minimieren.
\end{abstract}

\section{Einleitung}

Das Dengue-Fieber ist eine infektiöse Virenerkrankung, die durch den Stich von weiblichen Moskitos der Spezies Aedes auf den Menschen übertragen wird [1]. Eine direkte Mensch-zu-Mensch-Infektion ist nicht möglich. Aus serologischer Sicht gehört der Dengue-Virus zur Familie des Flavivirus und es sind vier verschiedene Serotypen DENV-1, DENV-2, DENV-3 und DENV4 bekannt. Bei einer Infektion werden nur Antikörper gegen den verursachenden Serotypen gebildet. Es entsteht dadurch keine lebenslange, sondern nur eine temporäre Kreuzimmunität gegen die anderen DengueSerotypen [1]. Eine Erstinfektion verursacht meist nur grippeähnlichen Symptome, wie Schüttelfrost oder Gliederschmerzen. Eine Erkrankung an einem weiteren Serotypen führt zu einer höheren Viruslast, da die nach einer erstmaligen Infektion gebildeten Antikörper zusammen mit dem Virus des neuen Seroypen AntigenAntikörper-Viren-Komplexe bilden [2]. Infolgedessen sind Zweitinfektionen mit deutlich mehr Komplikationen verbunden, symptomatisch sind Atemnot oder Magen-Darm-Blutungen. Heutzutage ist die Krankheit in 128 Ländern als endemisch eingeordnet, wobei insbesondere die Region Asien/Pazifik betroffen ist [3]. Im Jahr 2014 wurden erstmalig Funde erwachsener Tigermücken im Süden Deutschlands nachgewiesen [4, 5]. In Deutschland wurden im Jahr 2018 über 600 Infektionen mit Dengue registriert, die durch Rückreisende aus Risikogebieten der Tropen verursacht wurden [6]. Das benutzte Modell für Dengue-Fieber basiert auf Untersuchungen in [7, 8, 9]. In dieser Ausarbeitung wird ein Zwei-Serotypen-Modell für DengueFieber untersucht, welches auf mehreren Überlegungen beruht $[10,11,12,13]$.

\section{Modell mit temporärer Kreuzimmunität und Kontrollmaßnahmen}

Mit 1 und 2 werden im folgenden zwei verschiedene, aber fest gewählte, der vier möglichen DengueSerotypen DENV-1, DENV-2, DENV-3 und DENV-4 beschrieben. Für die beiden Indizes $i, j$, mit $i \neq j$, gelte stets $i, j \in\{1,2\}$.

Das SIR-Modell stellt die menschliche Population, welche in zehn Kompartimente unterteilt wird, dar. $S_{h}$ entspricht den für beide Serotypen anfälligen Individuen, $I_{h}^{i}$ den Erstinfizierten mit dem Serotyp $i, R_{h}^{i}$ den gegen den Serotyp $i$ resistenten Individuen, $S_{h}^{i}$ entspricht den für Serotyp $i$ anfälligen Individuen, $I_{h}^{j i}$ den Zweitinfizierten mit Serotyp $i$ und $R_{h}$ den gegen beide Seroty- 
pen resistenten Individuen. Die Moskitopopulation, untergliedert in vier Kompartimente, wird durch das ASIModell repräsentiert. $A_{m}$ beschreibt die Vektoren in der aquatischen Phase, $S_{m}$ die gegen den Virus anfällige Stechmücken und $I_{m}^{i}$ die mit Serotyp $i$ infizierten Moskitos, welche infizierend sind.

Für die Modellierung der Differentialgleichungen müssen noch weitere Annahmen getroffen werden [7, 9]. Beide Populationen werden als homogen angenommen, die räumlich gleichmäßig verteilt sind. Somit ist die Wahrscheinlichkeit, sich mit einem Serotypen zu infizieren, für jedes Individuum gleich groß. Bereits infizierte Individuen können nicht gleichzeitig an einem weiteren Serotypen erkranken. Des Weiteren wird vorausgesetzt, dass nur zwei der vier Dengue-Serotypen sowohl in der Bevölkerung als auch in der Vektorpopulation präsent sind. Da für die menschliche Population $N_{h}(t)$ keine Migration berücksichtigt wird, ist diese konstant für alle Zeiten $t$ und die einzelnen Kompartimente sind als stetige Größen zu betrachten. Insofern ist die Größe der Bevölkerung unabhängig von $t$ und es muss gelten:

$$
\begin{aligned}
& N_{h}=S_{h}(t)+I_{h}^{1}(t)+I_{h}^{2}(t)+R_{h}^{1}(t)+R_{h}^{2}(t)+ \\
& +S_{h}^{1}(t)+S_{h}^{2}(t)+I_{h}^{12}(t)+I_{h}^{21}(t)+R_{h}(t) \quad \forall t
\end{aligned}
$$

Durch den Proportionalitätsfaktor $\mu_{h}$ wird die natürliche Sterberate der Menschen beschrieben, der dazugehörige Kehrwert stellt die durchschnittliche Lebenserwartung der menschlichen Population dar. Bei einer Erkrankung mit einem Dengue-Virus wird keine erhöhte Sterblichkeitsrate angenommen. Damit die Bevölkerungszahl konstant bleibt, gibt es zum Kompartiment $S_{h}$ einen konstanten Zufluss $\mu_{h} N_{h}$, der die Neugeborenen repräsentiert. Diese sind gesund, aber anfällig für eine Infektion mit einem der beiden Serotypen. Die Parameter, welche die Übertragung des Dengue-Virus beeinflussen, w erden $n$ achfolgend $b$ eschrieben. $D$ ie durchschnittliche Stechrate der Moskitos pro Tag ist definiert durch den Parameter $B$. Die Wahrscheinlichkeit einer Übertragung beim Stich eines Menschen, verursacht durch Aedes-Stechmücken, wird durch $\beta_{m h}$ beschrieben. Bei einer Infektion mit dem Dengue-Virus wird die Genesungsrate durch den Faktor $\eta_{h}$ definiert. Dessen Kehrwert charakterisiert in Tagen die durchschnittliche Erkrankungsdauer der Menschen. Der Kehrwert des neu hinzugefügten Parameters $\zeta$ stellt die Dauer der temporären Kreuzimmunität nach der Erkrankung mit einem Serotypen dar. Analog zum Parameter $\mu_{h}$ wird die natürliche Sterberate der ausgewachsenen Vektorpopulation durch den Proportionalitätsfaktor $\mu_{m}$ dargestellt. Ihr Kehrwert entspricht der durchschnittlichen Lebenserwartung der Moskitos. Die Sterberate der nicht ausgewachsenen Moskitos wird durch den Parameter $\mu_{A}$ beschrieben. Analog zu den Neugeborenen sind diese bei Geburt gesund. $\eta_{A}$ beschreibt in Tagen die durchschnittliche Dauer der Vektoren in der aquatischen Phase. Der Kehrwert des Faktors ist als Reifungsrate der Larven zu ausgewachsenen Moskitos zu verstehen. Es wird vorausgesetzt, dass jeder ausgewachsene Moskito im Durchschnitt täglich $\varphi$ Eier in einen Brutplatz legt. Diese Brutplätze sind bezüglich der Anzahl der Eier beschränkt, was durch die Trägerkapazität $k N_{h}$ ersichtlich wird. Der Faktor $k$ beschreibt die durchschnittliche Anzahl an nicht-ausgewachsenen Stechmücken pro Mensch. Sind die Moskitos ausgereift, so verlassen diese die aquatische Phase und können sich nun mit einem Dengue-Virus infizieren. Analog zur aquatischen Phasen ist auch die Anzahl der ausgewachsenen Moskitos beschränkt, dargestellt durch den Faktor $m$. Die Übertragungswahrscheinlichkeit von Mensch zu Moskitos ist durch den Parameter $\beta_{h m}$ definiert. Die zuvor beschriebenen Parameter sind positiv und die Eintrittswahrscheinlichkeiten sind zusätzlich nach oben durch 1 beschränkt.

Weiterhin werden in das Modell drei Kontrollmöglichkeiten eingefügt:

Anteil an Larvizid:

Anteil an Adultizid:

$$
\begin{aligned}
& 0 \leq c_{A}(t) \leq 1 \\
& 0 \leq c_{m}(t) \leq 1
\end{aligned}
$$$$
\text { Anteil an mech. Kontrolle: } \quad 0<\alpha_{\min } \leq \alpha(t) \leq 1
$$

Mit mechanischer Kontrolle wird die Anzahl der Brutplätze der Moskitos reduzieren, beispielsweise Wasseransammlungen in Blumenuntersetzern, Moskitonetze über Regentonnen, Reduzierung von Altreifen zur Abdeckung von Silofolien in der Landwirtschaft usw. Zudem versteht man darunter auch die persönliche Vorbeugung, wie das Tragen von langen Hosen und Ärmeln, und das Anbringen von Fliegengittern an Fenstern und Türen. Das Verteilen von Lariviziden wird zur Bekämpfung der aquatischen Stechmücken eingesetzt, das Sprühen von Adultiziden zur Reduzierung der erwachsenen Moskitopulation.

Unter Berücksichtigung der beschriebenen Sachverhalte entsteht ein Kompartiment-Modell, das in Abbildung 1 skizziert ist. Die Kompartimente der anfälligen Population sind blau, die der Infizierten rot und die der resistenten Population grün dargestellt. 


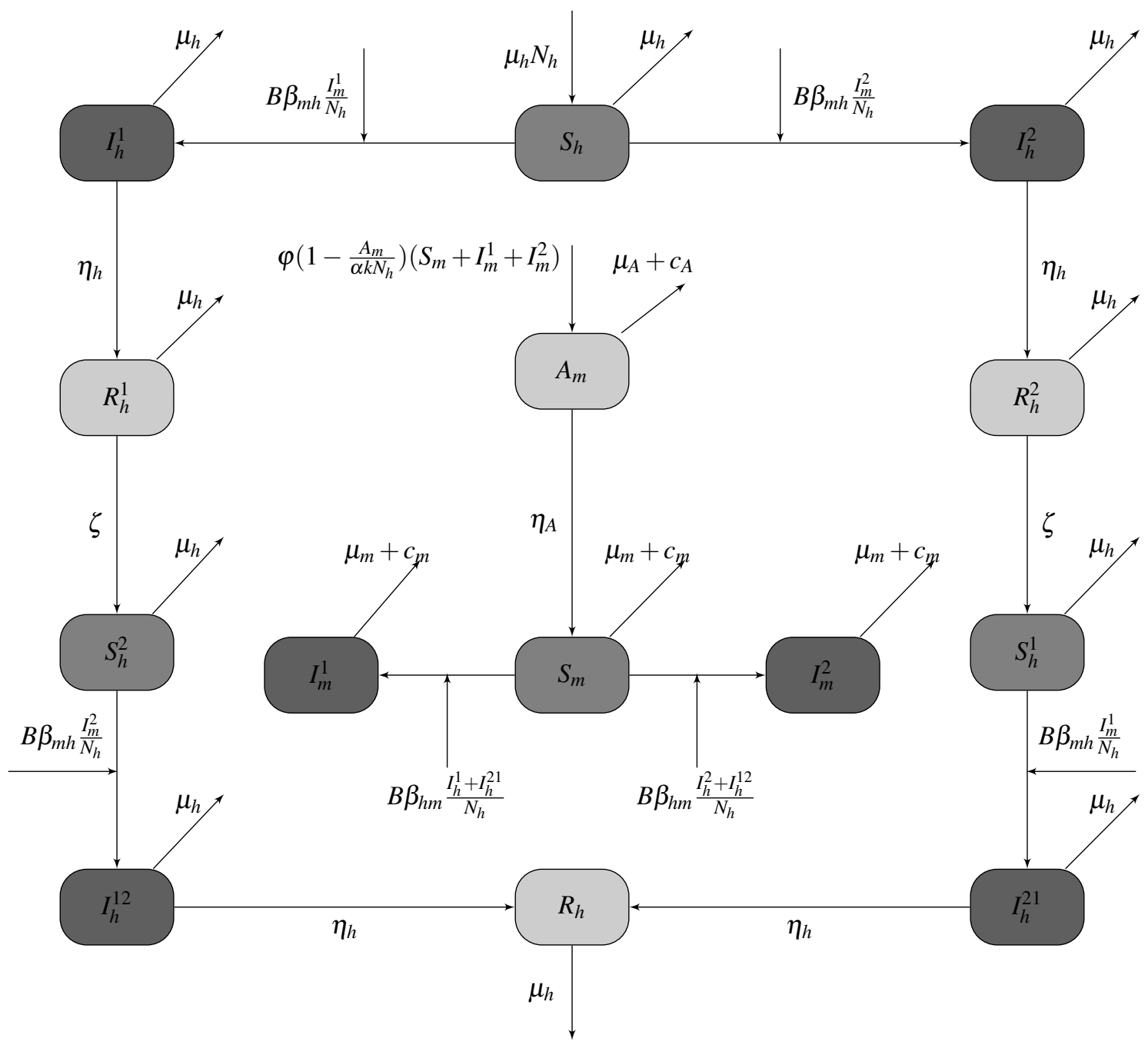

Abbildung 1: Kompartimentmodell mit temporärer Kreuzimmunität

Zusammenfassend ergibt sich ein System von nichtlinearen Differentialgleichungen. Dabei gilt für die menschliche Population

$$
\left\{\begin{array}{l}
\left.\dot{S}_{h}=\mu_{h} N_{h}-\left(B \beta_{m h} \frac{I_{m}^{1}+I_{m}^{2}}{N_{h}}\right)+\mu_{h}\right) S_{h} \\
\dot{I}_{h}^{i}=B \beta_{m} \frac{I_{m}^{i}}{N_{h}} S_{h}-\left(\eta_{h}+\mu_{h}\right) I_{h}^{i} \\
\dot{R}_{h}^{i}=\eta_{h} I_{h}^{i}-\left(\zeta+\mu_{h}\right) R_{h}^{i} \\
\dot{S}_{h}^{i}=\zeta R_{h}^{j}-\left(B \beta_{m h} \frac{I_{m}^{i}}{N_{h}}+\mu_{h}\right) S_{h}^{i} \\
\dot{I}_{h}^{i j}=B \beta_{m h} \frac{I_{m}^{j}}{N_{h}} S_{h}^{j}-\left(\eta_{h}+\mu_{h}\right) I_{h}^{i j} \\
\dot{R}_{h}=\eta_{h}\left(I_{h}^{12}+I_{h}^{21}\right)-\mu_{h} R_{h}
\end{array}\right.
$$

und für die Moskitopopulation

$$
\left\{\begin{array}{l}
\dot{A}_{m}=\varphi\left(1-\frac{A_{m}}{\alpha k N_{h}}\right)\left(S_{m}+I_{m}^{1}+I_{m}^{2}\right)- \\
-\left(\eta_{A}+\mu_{A}+c_{A}\right) A_{m} \\
\dot{S}_{m}=\eta_{A} A_{m}-\left(B \beta_{h m} \frac{I_{h}^{1}+I_{h}^{2}+I_{h}^{12}+I_{h}^{21}}{N_{h}}+\mu_{m}+c_{m}\right) S_{m} \\
\dot{I}_{m}^{i}=B \beta_{h m} \frac{I_{h}^{i}+I_{h}^{j i}}{N_{h}} S_{m}-\left(\mu_{m}+c_{m}\right) I_{m}^{i}
\end{array}\right.
$$

mit $i, j \in\{1,2\}, i \neq j$.

\section{Theoretische Analyse}

Die Steuerungen $c_{A}, c_{m}$ und $\alpha$ werden für die theoretische Analyse als konstante Größen angenommen. Diese 
wird auf der nachfolgenden Menge durchgeführt:

$$
\begin{gathered}
\Omega=\left\{\left(S_{h}, I_{h}^{1}, I_{h}^{2}, R_{h}^{1}, R_{h}^{2}, S_{h}^{1}, S_{h}^{2}, I_{h}^{12}, I_{h}^{21}, R_{h} \mid\right.\right. \\
\left.A_{m}, S_{m}, I_{m}^{1}, I_{m}^{2}\right) \in \mathbb{R}_{+}^{14} \mid \\
S_{h}+R_{h}+\sum_{i}\left(I_{h}^{i}+R_{h}^{i}+S_{h}^{i}\right)+\sum_{i, j} I_{h}^{i j} \leq N_{h}, \\
\left.A_{m} \leq k N_{h}, S_{m}+I_{m}^{1}+I_{m}^{2} \leq m N_{h}\right\}
\end{gathered}
$$

Die einzelnen Kompartimente, die die menschliche Population beschreiben, dürfen in der Summe die Gesamtbevölkerung nicht überschreiten. Zudem sind die Anzahl der Larven und die Summe der ausgewachsenen Moskitos durch die menschliche Bevölkerungszahl beschränkt.

Satz 1 : Für das DGL-System (1), (2) ist die Menge $\mathbb{R}_{+}^{14}$ und insbesondere auch $\Omega$ positiv invariant.

Beweis: Das DGL-System lässt sich für $X \in \mathbb{R}_{+}^{14}$, $X=\left(S_{h}, I_{h}^{1}, I_{h}^{2}, R_{h}^{1}, R_{h}^{2}, S_{h}^{1}, S_{h}^{2}, I_{h}^{12}, I_{h}^{21}, R_{h} \mid A_{m}, S_{m}, I_{m}^{1}, I_{m}^{2}\right)$, in ein Metzler-System $\dot{X}=A(X) X+F$ umschreiben. Es gilt $F=\left(\mu_{h} N_{h}, 0,0,0,0,0,0,0,0,0 \mid 0,0,0,0\right)^{T}$ und $A(X)=\left(\begin{array}{cc}A_{h}(X) & 0 \\ 0 & A_{m}(X)\end{array}\right)$, Details siehe Figur 2.

Die Blockdiagonalmatrix $A(X)$ ist eine MetzlerMatrix, da die Werte auf der Diagonalen negativ sind und die Einträge außerhalb der Diagonalen nur nichtnegativ sind. Ferner gilt $F \geq 0$. Nach den Ausführungen in $[14,15]$ sind $\mathbb{R}_{+}^{14}$ und $\Omega$ positiv invariant.

Nach diesem Resultat lässt sich festhalten, dass die Lösungen für alle Startwerte aus $\Omega$ innerhalb der Menge $\Omega$ verlaufen. Dementsprechend können bei der Wahl eines biologisch sinnvollen Startwerts keine biologisch nicht-relevanten Lösungen entstehen.

Die nachfolgenden Lösungen wurden mit dem Computeralgebrasystem Maple berechnet. Zur besseren Darstellung werden folgende Hilfsgrößen eingefügt:

$$
\begin{gathered}
\mathscr{A}_{\text {kontroll }}=\varphi \eta_{A}-\left(\mu_{m}+c_{m}\right)\left(\eta_{A}+\mu_{A}+c_{A}\right), \\
\mathscr{B}_{\text {kontroll }}=\alpha k B^{2} \beta_{h m} \beta_{m h} \mathscr{A}_{\text {kontroll }}, \\
\mathscr{C}_{\text {kontroll }}=\varphi\left(\mu_{m}+c_{m}\right)^{2}\left(\eta_{h}+\mu_{h}\right), \\
\mathscr{D}_{\text {kontroll }}=B \beta_{\text {hm }}\left(\alpha B k \beta_{\text {mh }} \mathscr{A}_{\text {kontroll }}+\varphi \mu_{h}\left(\mu_{m}+c_{m}\right)\right) .
\end{gathered}
$$

Satz 2 : In $\Omega$ besitzt das DGL-System (1), (2) bis zu sechs Gleichgewichtspunkte, davon vier Gleichgewichte mit aussterbenden Serotypen. Maximal zwei sind krankheitsfrei $\left(E_{1}^{*}, E_{2}^{*}\right)$ und höchstens zwei sind endemische Randgleichgewichte $\left(E_{3}^{*}, E_{4}^{*}\right)$. Diese nehmen folgende Werte an:

$$
\begin{aligned}
- & E_{1}^{*}=\left(N_{h}, 0,0,0,0,0,0,0,0,0 \mid 0,0,0,0\right) \\
\text { - } & E_{2}^{*}=\left(N_{h}, 0,0,0,0,0,0,0,0,0 \mid\right. \\
& \left.\frac{k N_{h} \otimes A_{\text {kontroll }}}{\varphi \eta_{A}}, \frac{k N_{h} \otimes \otimes_{\text {kontroll }}}{\varphi\left(\mu_{m}+c_{m}\right)}, 0,0\right)
\end{aligned}
$$

$$
\begin{aligned}
& \text { - } E_{3}^{*}=\left(S_{h}^{* *}, I_{h}^{1 * *}, 0, R_{h}^{1 * *}, 0,0, S_{h}^{2 * *}, 0,0,0 \mid\right. \\
& \left.A_{m}^{* *}, S_{m}^{* *}, I_{m}^{1 * *}, 0\right) \\
& \text { - } E_{4}^{*}=\left(S_{h}^{* *}, 0, I_{h}^{2 * *}, 0, R_{h}^{2 * *}, S_{h}^{1 * *}, 0,0,0,0 \mid\right. \\
& \left.A_{m}^{* *}, S_{m}^{* *}, 0, I_{m}^{2 * *}\right), \text { mit } \\
& \text { - } S_{h}^{* *}=\frac{N_{h}\left(B \beta_{h m} \varphi \mu_{h}\left(\mu_{m}+c_{m}\right)+\mathscr{C}_{\text {kontroll }}\right)}{\mathscr{D}_{\text {kontroll }}} \\
& \text { - } I_{h}^{i * *}=\frac{\mu_{h} N_{h}\left(\mathscr{B}_{\text {kontroll }}-\mathscr{C}_{\text {kontroll }}\right)}{\left(\eta_{h}+\mu_{h}\right) \mathscr{D}_{\text {kontroll }}} \\
& \text { - } R_{h}^{i * *}=\frac{\eta_{h} \mu_{h} N_{h}\left(\mathscr{B}_{\text {kontroll }}-\mathscr{C}_{\text {kontroll }}\right)}{\left(\zeta+\mu_{h}\right)\left(\eta_{h}+\mu_{h}\right) \mathscr{D}_{\text {kontroll }}} \\
& \text { - } S_{h}^{i * *}=\frac{\zeta \eta_{h} N_{h}\left(\mathscr{B}_{\text {kontroll }}-\mathscr{C}_{\text {kontroll }}\right)}{\left(\zeta+\mu_{h}\right)\left(\eta_{h}+\mu_{h}\right) \mathscr{D}_{\text {kontroll }}} \\
& \text { - } A_{m}^{* *}=\frac{k N_{h} \mathscr{A}_{\text {kontroll }}}{\varphi \eta_{A}} \\
& \text { - } S_{m}^{* *}=\frac{\left(\mu_{h}+\eta_{h}\right) N_{h}\left(\alpha k B \beta_{m h} \mathscr{S k}_{\text {kontroll }}+\varphi \mu_{h}\left(\mu_{m}+c_{m}\right)\right)}{B \beta_{m h} \varphi\left(B \beta_{h m} \mu_{h}+\left(\eta_{h}+\mu_{h}\right)\left(\mu_{m}+c_{m}\right)\right)} \\
& \text { - } I_{m}^{i * *}=\frac{\mu_{h} N_{h}\left(\mathscr{B}_{\text {kontroll }}-\mathscr{C}_{\text {kontroll }}\right)}{B \beta_{m h}\left(\mathscr{C}_{\text {kontroll }}+B \beta_{h m} \varphi \mu_{h}\left(\mu_{m}+c_{m}\right)\right)}
\end{aligned}
$$

Bemerkungen: Der Punkt $E_{1}^{*}$ beschreibt einen trivialen Gleichgewichtspunkt, während $E_{2}^{*}$ ein nichttriviales, krankheitsfreies Equilibrium (kurz: DFE) darstellt. In beiden Punkten gibt es keine infizierte Menschen und Moskitos sowie keine gegen mindestens einen Serotypen resistente Menschen.

Satz 3 : Die Basisreproduktionszahl $\mathscr{R}_{0}$ des DGLSystems (1), (2) erfüllt:

$$
\mathscr{R}_{0}=\sqrt{\frac{\mathscr{B}_{\text {kontroll }}}{\mathscr{C}_{\text {kontroll }}}}=\sqrt{\frac{\alpha k B^{2} \beta_{\text {hm }} \beta_{\text {mh }} \mathscr{A}_{\text {kontroll }}}{\varphi\left(\mu_{m}+c_{m}\right)^{2}\left(\eta_{h}+\mu_{h}\right)}}
$$

Beweis: Zur Berechnung der Basisreproduktionszahl (kurz: BRN) wird das Next-Generation-Verfahren angewandt $[16,17,18,19]$ zu finden. Mit dieser Methode nach [18] lässt sich für jeden Serotypen $i$, mit $i \in\{1,2\}$, eine BRN bestimmen. In die Berechnungen fließen nur Kompartimente mit Neuinfektionen ein. Die Rate von Neuinfektion mit Serotyp $i$ wird durch den Vektor $\mathscr{F}_{i}$ dargestellt, $\mathscr{V}_{i}$ umfasst die restlichen Transferterme bezüglich Serotyp $i$, d.h. Tode, Geburten, Krankheitsveränderungen sowie Heilungen. Die in dieser Ausarbeitung vorgenommene Zerlegung ist nach $[16,18]$ gewählt, aufgrund verschiedener Deutungen im Krankheitsverlauf ist diese aber nicht zwingend eindeutig. Von den zuvor beschriebenen Vektoren werden die Jacobi-Matrizen berechnet, die mit $F_{i}$ beziehungsweise $V_{i}$ bezeichnet werden. Diese werden am nichtrivialen, krankheitsfreien Gleichgewicht $E_{2}^{*}$ ausgewertet. Dabei ist $F_{i}$ eine nicht-negative Matrix und $V_{i}$ eine invertierbare M-Matrix. Abschließend wird das Matrixprodukt $F_{i} V_{i}^{-1}$ betrachtet, welches als Next-Generation-Matrix bezeichnet wird. Dessen Spektralradius entspricht der 


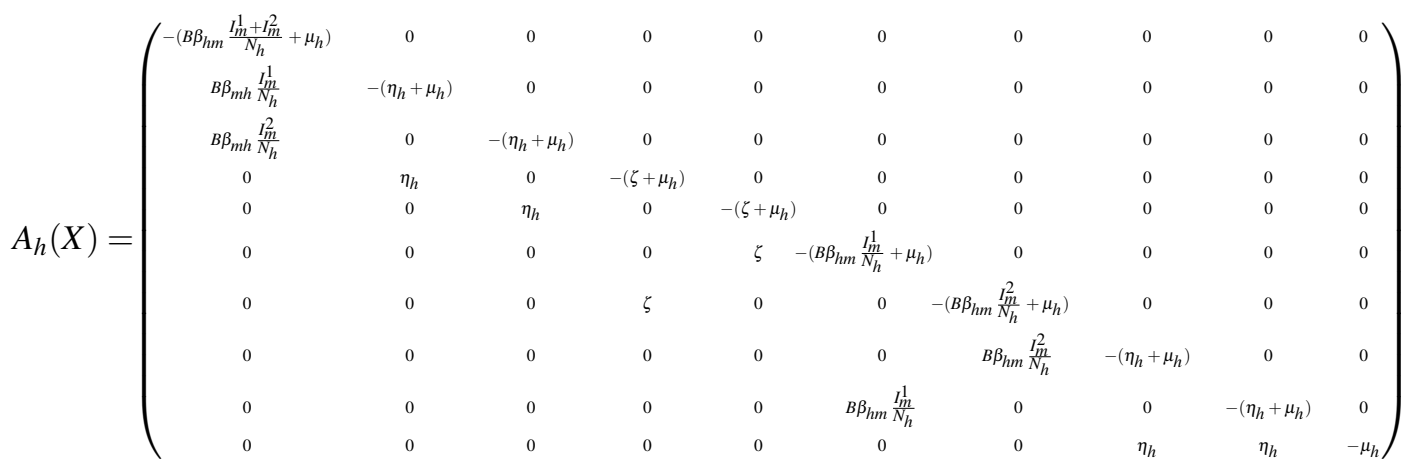

$$
\begin{aligned}
& A_{m}(X)=\left(\begin{array}{cccc}
-\left(\varphi \frac{S_{m}+l_{m}^{1}+l_{m}^{2}}{\alpha k N_{h}}+\eta_{A}+\mu_{A}+c_{A}\right) & \varphi & \varphi & \varphi \\
\eta_{A} & -\left(B \beta_{h m} \frac{l_{h}^{1}+l_{h}^{2}+I_{h}^{12}+l_{h}^{21}}{N_{h}}+\mu_{m}+c_{m}\right) & 0 & 0 \\
0 & B \beta_{h m} \frac{I_{h}^{1}+l_{h}^{21}}{N_{h}} & -\left(\mu_{m}+c_{m}\right) & 0 \\
0 & B \beta_{h m} \frac{I_{h}^{2}+I_{h}^{12}}{N_{h}} & 0 & -\left(\mu_{m}+c_{m}\right)
\end{array}\right)
\end{aligned}
$$

Abbildung 2: Teilmatrizen von $A(X)$

BRN des Serotypen $i$, d.h. es gilt: $\mathscr{R}_{i}=\rho\left(F V^{-1}\right)$. Untersuchungen in [19] haben gezeigt, dass das DFE nicht eindeutig sein muss.

Für Serotyp 1 mit den Kompartimenten $I_{h}^{1}, I_{h}^{21}$ und $I_{m}^{1}$ lassen sich die folgenden Vektoren bilden:

$$
\mathscr{F}_{1}=\left(\begin{array}{c}
B \beta_{m h} \frac{I_{m}^{1}}{N_{h}} S_{h} \\
B \beta_{m h} \frac{I_{m}^{1}}{N_{h}} S_{h}^{1} \\
B \beta_{h m} \frac{I_{h}^{1}+I_{h}^{21}}{N_{h}} S_{m}
\end{array}\right), \mathscr{V}_{1}=\left(\begin{array}{c}
\left(\eta_{h}+\mu_{h}\right) I_{h}^{1} \\
\left(\eta_{h}+\mu_{h}\right) I_{h}^{21} \\
\left(\mu_{m}+c_{m}\right) I_{m}^{2}
\end{array}\right) .
$$

Nach Einsetzen der Werte des Gleichgewichts $E_{2}^{*}$ sehen die jeweiligen Jacobi-Matrizen $F_{1}$ und $V_{1}$ wie folgt aus:

$$
\begin{gathered}
F_{1}=\left(\begin{array}{ccc}
0 & 0 & B \beta_{m h} \frac{S_{h}^{*}}{N_{h}} \\
0 & 0 & B \beta_{m h} \frac{S_{h}^{1 *}}{N_{h}} \\
B \beta_{h m} \frac{S_{m}^{*}}{N_{h}} & B \beta_{h m} \frac{S_{m}^{*}}{N_{h}} & 0
\end{array}\right), \\
V_{1}=\left(\begin{array}{ccc}
\eta_{h}+\mu_{h} & 0 & 0 \\
0 & \eta_{h}+\mu_{h} & 0 \\
0 & 0 & \mu_{m}+c_{m}
\end{array}\right)
\end{gathered}
$$

Die Next-Generation-Matrix $F_{1} V_{1}^{-1}$ nimmt somit folgende Gestalt an:

$$
F_{1} V_{1}^{-1}=\left(\begin{array}{ccc}
0 & 0 & \frac{B \beta_{m h}}{\mu_{m}+c_{m}} \\
0 & 0 & 0 \\
\frac{\alpha k B \beta_{h m} \otimes A_{\text {kontroll }}}{\varphi\left(\mu_{m}+c_{m}\right)\left(\eta_{h}+\mu_{h}\right)} & \frac{\alpha k B \beta_{h m} \alpha A_{\text {knntroll }}}{\varphi\left(\mu_{m}+c_{m}\right)\left(\eta_{h}+\mu_{h}\right)} & 0
\end{array}\right)
$$

Die BRN des Serotyps 1 berechnet sich zu:

$$
\mathscr{R}_{1}=\rho\left(F_{1} V_{1}^{-1}\right)=\sqrt{\frac{\alpha k B^{2} \beta_{h m} \beta_{m h} \mathscr{A}_{\text {kontroll }}}{\varphi\left(\mu_{m}+c_{m}\right)^{2}\left(\eta_{h}+\mu_{h}\right)}}
$$

Aufgrund der symmetrischen Betrachtungsweise ergibt die Berechnung für die BRN des Serotyps 2 das gleiche Ergebnis. Somit folgt:

$$
\mathscr{R}_{2}=\rho\left(F_{2} V_{2}^{-1}\right)=\sqrt{\frac{\alpha k B^{2} \beta_{h m} \beta_{m h} \mathscr{A}_{\text {kontroll }}}{\varphi\left(\mu_{m}+c_{m}\right)^{2}\left(\eta_{h}+\mu_{h}\right)}}
$$

Es gilt $\mathscr{R}_{1}=\mathscr{R}_{2}$ und die Behauptung in (3) folgt aus $\mathscr{R}_{0}=\max \left\{\mathscr{R}_{1}, \mathscr{R}_{2}\right\}$.

Analog zu [20] kann die BRN wie folgt umgeschrieben werden:

$$
\begin{aligned}
\mathscr{R}_{0}^{2}= & \frac{\mathscr{B}_{\text {kontroll }}}{\mathscr{C}_{\text {kontroll }}}=\frac{\alpha k B^{2} \beta_{h m} \beta_{\text {mh }} \mathscr{A}_{\text {kontroll }}}{\varphi\left(\mu_{m}+c_{m}\right)^{2}\left(\eta_{h}+\mu_{h}\right)}= \\
& =B^{2} \beta_{h m} \beta_{m h} \cdot \frac{\mathscr{E}_{m}}{N_{h}} \cdot \frac{\alpha}{\mu_{m}+c_{m}} \cdot \frac{1}{\eta_{h}+\mu_{h}},
\end{aligned}
$$

wobei $\mathscr{E}_{m}=S_{m}^{*}=\frac{\eta_{A}}{\mu_{m}+c_{m}} A_{m}^{*}=\frac{k N_{h} \mathscr{A k}_{\text {kontroll }}}{\varphi\left(\mu_{m}+c_{m}\right)}$ gewählt wurde.

Die ersten drei Terme repräsentieren die Stichintensität. Der zweite Ausdruck gibt die Anzahl der erwachsenen Stechmücken pro Mensch an. Der anschließende Ausdruck ist abhängig von den Parametern der Moskitos sowie der Kontrollmaßnamen. Abschließend folgt ein Term in Abhängigkeit der menschlichen Parameter.

Anhand dieser Kennzahl ist zu beobachten, dass eine erhöhte Infektionsrate bei einem Stich, sowohl von 
Mensch als auch von Moskito ( $\left.\beta_{h m}, \beta_{m h} \nearrow\right)$, die BRN $\mathscr{R}_{0}$ vergrößert. Ein ähnlicher Effekt ist bei einer erhöhten täglichen Stechrate $(B \nearrow)$ zu beobachten. Durch eine künstliche Erhöhung der Sterblichkeitsrate der Moskitos $\left(\mu_{m} \nearrow\right)$, das einer Minimierung der Lebensdauer der Stechmücken entspricht, sinkt $\mathscr{R}_{0}$.

Die Basisreproduktionszahl steht im engen Zusammenhang mit der Stabilität des DFE und dient als Schwellenwertparameter, was im nachfolgenden Satz verdeutlicht wird.

Satz 4 [16]: Das krankheitsfreie Gleichgewicht $E_{2}^{*}$ ist lokal asymptotisch stabil, falls $\mathscr{R}_{0}<1$ gilt. Für $\mathscr{R}_{0}>$ 1 lässt sich Instabilität folgern.

\section{Numerische Simulation}

Ohne Kontrollmaßnahmen wird eine numerische Simulation durchgeführt, welche die Lösungen der Kompartimente im zeitlichen Verlauf grafisch darstellt. Die Berechnung wurde in Matlab mit der Routine ode45 durchgeführt, die verwendeten Parameter stammen aus [21]. In diesen Ausführungen sind Grafiken für die Parameter der Steckmücke Aedes aegypti und für die Übertragung des Dengue-Virus in Abhängigkeit der Umgebungstemperatur abgebildet. Wie im asymmetrischen Modell ohne temporäre Kreuzimmunität in [22] wurden die Werte bei einer Temperatur von $30^{\circ} \mathrm{C}$ übernommen. Der Parameter $m=\frac{k \eta_{A}}{\mu_{m}}$, der die Anzahl der Moskitos pro Mensch abbildet, bildet sich aus denen im Ausdruck dargestellten Parametern. Zusammengefasst nehmen diese nachfolgende Werte an:

$$
\begin{gathered}
N_{h}=386000, B=0.22, \beta_{h m}=0.66, \beta_{m h}=0.78, \\
\eta_{A}=0.12, \eta_{h}=\frac{1}{3}, \mu_{A}=0.18, \\
\mu_{h}=\frac{1}{80 \cdot 365}, \mu_{m}=\frac{1}{23}, \varphi=8, k=3, m=8.28 .
\end{gathered}
$$

Der Anteil der infizierten Population liegt zu Beginn bei $0.01 \%$, welcher sich gleichmäßig auf beide Serotypen aufteilt. Die Startwerte der ausgewachsenen sowie nicht ausgewachsenen Vektoren entsprechen den jeweiligen Trägerkapazitäten. Es ergeben sich die folgenden Anfangswerte:

$$
\begin{gathered}
S_{h 0}=99.99 \% N_{h}, I_{h 0}^{1}=I_{h 0}^{2}=0.005 \% N_{h}, \\
R_{h 0}^{1}=R_{h 0}^{2}=S_{h 0}^{1}=S_{h 0}^{2}=I_{h 0}^{12}=I_{h 0}^{21}=R_{h 0}=0, \\
A_{m 0}=k N_{h}, S_{m 0}=m N_{h}, I_{m 0}^{1}=I_{m 0}^{2}=0 .
\end{gathered}
$$

Für die numerische Simulation wurde $\zeta=\frac{2}{365}$ gesetzt, sodass eine temporäre Kreuzimmunität mit einer Dauer von sechs Monaten angenommen wird.

Nach Übernehmen der Parameterdaten nehmen die Gleichgewichte die folgenden Werte an:

- $E_{1}^{*}=(386000,0,0,0,0,0,0,0,0,0 \mid 0,0,0,0)$

- $E_{2}^{*}=(386000,0,0,0,0,0,0,0,0,0, \mid$ $1142266,3152655,0,0)$

- $E_{3}^{*}=(13000,38,0,2316,0,0,370644,0,0,0 \mid$ $1142266,3151135,1519,0)$

- $E_{4}^{*}=(13000,0,38,0,2316,370644,0,0,0,0 \mid$ $1142266,3151135,0,1519)$

Für die BRN folgt: $\mathscr{R}_{0}=5.45>1$. Folglich ist das krankheitsfreie Gleichgewicht $E_{2}^{*}$ instabil und das Dengue-Virus wird sich langfristig innerhalb der Population verbreiten.

In der Figur 3 ist der Lösungsverlauf der infizierten Kompartimente dargestellt. Aufgrund der symmetrischen Betrachtungsweise sind die Lösungsverläufe für beide Serotypen identisch. In der linken Grafik, welche die Lösungen der infizierten Menschen abbildet, kommt es zwischen dem 10. und 40. Tag zu einem Krankheitsausbruch, in der Spitze bis zu etwa 60712 Menschen erkranken dabei erstmalig mit einem DengueSerotypen. Betrachtet man beide Serotypen, so sind maximal $31.5 \%$ der Gesamtbevölkerung gleichzeitig mit einem Serotyp infiziert. Danach werden keine weiteren Erstinfektionen mehr festgestellt. Die Lösungen der infizierten Stechmücken, abgebildet in der rechten Grafik, erreichen ihr Maximum während des Krankheitsausbruchs bei circa 545055, sodass gleichzeitig über eine Millionen Moskitos infiziert sind. Danach sinken die Zahlen zunächst steil, nach knapp 90 Tagen fällt die Lösungskurve wesentlich flacher ab. Von immenser Bedeutung ist auch die Betrachtung der Zweitinfektionen, da diese im Krankheitsbild wesentlich gefährlicher verlaufen [1]. Zwischen dem 30. und 70. Tag verläuft die Lösungskurve der Individuen mit einer zweiten Infektion auf einem nahezu konstanten Level und die Lösung erreicht ihr Maximum bei 2614. In der Summe sind somit bis zu 1.4\% der Bevölkerung zweimalig an einem Dengue-Serotypen erkrankt.

\section{Optimale Steuerung}

In diesem Abschnitt werden zeitabhängige Steuerungen betrachtet, welche auf einem Zeitgitter stückweise konstant modelliert werden. Für das im folgenden gewählte 

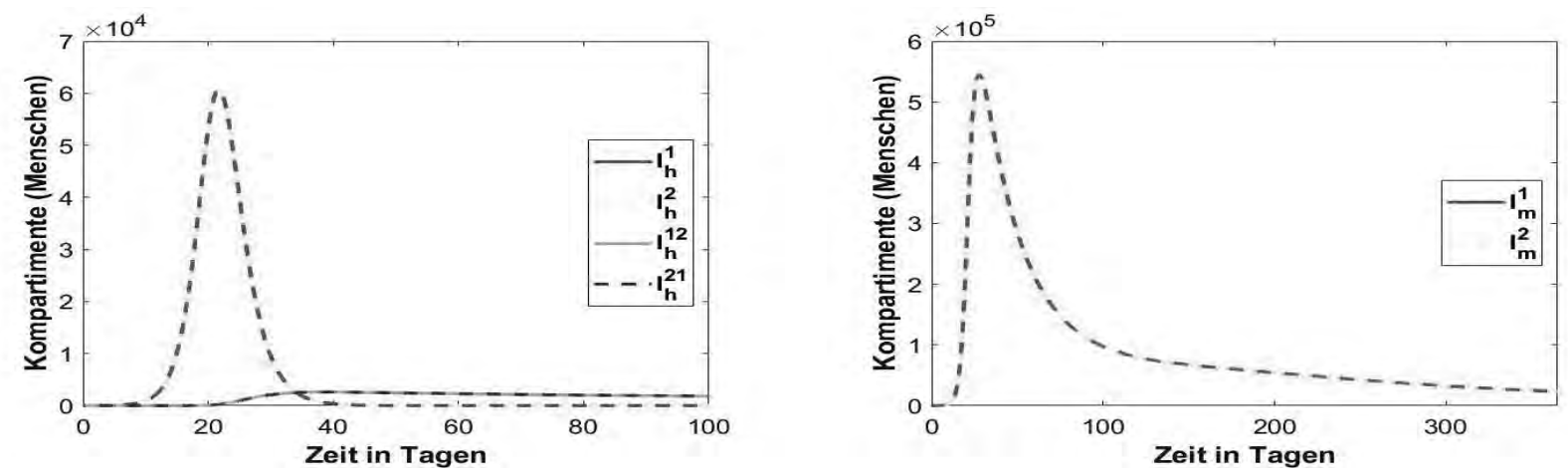

Abbildung 3: Lösungsverlauf für die infizierten Kompartimente der Menschen (links) und der Vektoren (rechts)

Kostenfunktional

$$
\begin{array}{r}
K=\int_{0}^{t_{f}}\left[\gamma_{D} \cdot i(t)^{2}+\gamma_{A} \cdot c_{m}(t)^{2}+\gamma_{L} \cdot c_{A}(t)^{2}+\right. \\
\left.+\gamma_{M} \cdot(1-\alpha(t))^{2}\right] \mathrm{d} t
\end{array}
$$

gilt $i(t)=\left[I_{h}^{1}(t)+I_{h}^{2}(t)+I_{h}^{12}(t)+I_{h}^{21}(t)\right] / N_{h}$ und es beinhaltet die Gewichte $\gamma_{j}$. Die obere Integralgrenze $t_{f}$ beträgt 365 [Tage] und die Anfangsbedingungen entsprechen denjenigen aus dem vorherigen Abschnitt. Die Kosten der verschiedenen Strategien zur Eindämmung der Moskitopopulation wurden mit der Modellierungssprache AMPL und dem Solver IPOPT berechnet $[23,24]$. Um numerisch stabile Ergebnisse zu erhalten, wurden das Zielfunktional sowie das System von Differentialgleichungen entdimensionalisiert. Drei verschiedene Fälle werden betrachtet: Ein mittleres Szenario (Fall A), teure Behandlungskosten für die infizierte Population (Fall B) sowie hohe Kosten für die Eindämmung der Stechmücken (Fall C).

Wie sich zeigt, führt eine längere, temporäre Kreuzimmunität und somit ein kleinerer Wert von $\zeta$ zu geringeren Kosten. Fall B, das eine möglichst geringe Anzahl an Infizierten priorisiert, ist mit relativ hohen Kosten verbunden. Fall $\mathrm{C}$, in dem die Eindämmung der Moskitos einen hohen Stellenwert besitzt, bildet den kostengünstigsten der drei Szenarien. In Fall A wird ein mittleres Szenario simuliert, die dazugehörigen Werte des Zielfunktionals liegen zwischen den beiden anderen Fällen.

In zwei Grafiken wird in Figur 4 der prozentuale Anteil an Erst- und Zweitinfektionen mit Serotyp 1 dargestellt. Aufgrund der Symmetrie im Modell gelten die nachfolgenden Zahlen für beide Serotypen und es genügt daher, nur einen Serotypen (hier: Serotyp 1) zu betrachten. Im mittleren Szenario (Fall A) treten ma- ximal $8.04 \%$ Erstinfektionen mit Serotyp 1 auf. Ohne Kontrollstrategien lagen diese Zahlen bei $15.7 \%$ für beide Serotypen, sodass die Anzahl der Primärinfizierten durch das Einbringen von Kontrolle nahezu halbiert werden konnte. Wird wieder Fall A (mittleres Szenario) betrachtet, so können bis zu $0.72 \%$ Zweitinfektionen registriert werden. Ohne Kontrolle lag diese Zahl bei $0.68 \%$, sodass die Anzahl der Sekundärinfizierten trotz Einsatz von Kontrollmaßnahmen einen leichten Anstieg verzeichnete.

In der Figur 5 ist der Einsatz der drei Kontrollstrategien im Betrachtungszeitraum abgebildet. Bei der mechanischen Kontrolle wird dabei die Differenz betrachtet, da für einen Wert nahe der 0 besonders viel Kontrolle ausgeübt wird. In allen drei Grafiken zeigt sich, dass im Szenario von teuren Behandlungskosten (Fall B) am meisten Kontrolle erbracht wird. Hier wird maximal bis $\mathrm{zu} 0.16 \%$ Larvizid, bis zu $12.83 \%$ Adultizid und bis $\mathrm{zu}$ $1.04 \%$ mechanische Kontrolle eingesetzt. Im Szenario mit teuren Kontrollmaßnahmen (Fall C) wird dementsprechend auch am wenigsten Kontrolle in einem kürzeren Zeitraum eingesetzt. Dabei werden bis zu $0.02 \%$ Larvizid, bis zu $3.74 \%$ Adultizid und bis zu $0.17 \%$ mechanische Kontrolle verwendet. Im mittleren Szenario (Fall A) liegen die Lösungskurven zwischen den zuvor beschriebenen Lösungen. Höchstens bis zu $0.05 \%$ Larvizid, bis zu $8.17 \%$ Adultizid und bis zu $0.50 \%$ mechanische Kontrolle wird eingesetzt. Nach spätestens 70 Tagen nehmen alle Lösungskurven ein relativ geringes konstantes Niveau, sodass nur noch sehr wenig Kontrolle ausgeübt wird. 


\begin{tabular}{c|c|c|c|c|} 
& Gewichte & \multicolumn{3}{|c|}{ Kosten } \\
\cline { 3 - 5 } & & $\zeta=\frac{1}{365}$ & $\zeta=\frac{2}{365}$ & $\zeta=\frac{4}{365}$ \\
\hline \hline A & $\gamma_{D}=\gamma_{A}=\gamma_{L}=\gamma_{M}=0.25$ & 0.119041 & 0.127501 & 0.142727 \\
\hline B & $\gamma_{D}=0.55, \gamma_{A}=\gamma_{L}=\gamma_{M}=0.15$ & 0.177034 & 0.196673 & 0.230429 \\
\hline C & $\gamma_{D}=0.10, \gamma_{A}=\gamma_{L}=\gamma_{M}=0.30$ & 0.057502 & 0.060873 & 0.066516
\end{tabular}

Tabelle 1: Gewichte und Kosten bei der Optimalen Steuerung
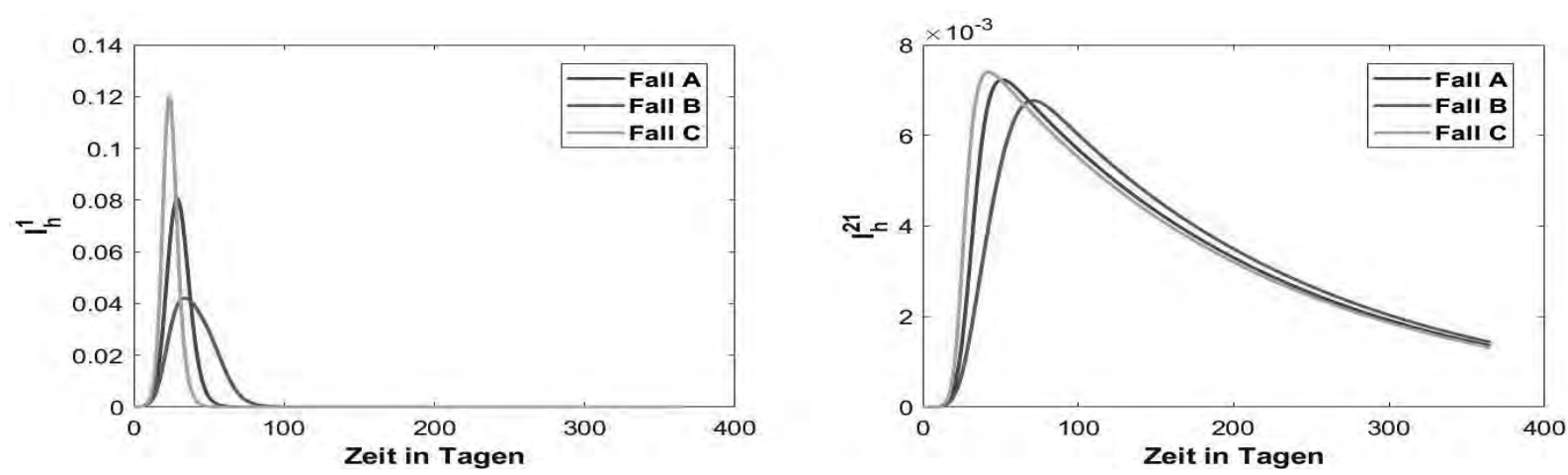

Abbildung 4: Erst- (links) und Zweitinfektionen (rechts) mit Serotyp 1

\section{Fazit}

Wie sich zeigt, ist das Einbringen von Kontrollstrategien eine sinnvolle Gegenmaßnahme, um die Anzahl der erstmalig infizierten Individuen zu minimieren und somit die Verbreitung des Virus einzudämmen. Als besonders effektiv stellt sich der Einsatz von Adultizid heraus, das zur Bekämpfung der erwachsenen Moskitopopulation eingesetzt wird. Allerdings erhöht sich durch diese Gegenmaßnahmen die maximale Anzahl der Zweitinfizierten, sodass in diesem Fall weitere Anpassungen der Strategien zwingend erforderlich sind. Zur Minimierung dieser Fallzahlen können auch zusätzliche geeignete Impfmaßnahmen in Betracht gezogen werden.

\section{Literatur}

[1] WHO. Dengue and severe dengue. September 2020.

[2] Recker M, Blyuss K, Simmons C, Tinh Tran H, Wills B, Farrar J, Gupta S. Immunological serotype interactions and their effect on the epidemiological pattern of dengue. Proceedings. Biological sciences / The Royal Society 05 2009; 276:2541-2548.

[3] Brady O, Gething P, Bhatt S, Messina J, Brownstein J, Hoen A, Moyes C, Farlow A, Scott T, Hay S. Refining the global spatial limits of dengue virus transmission by evidence-based consensus. PLoS neglected tropical diseases 08 2012; 6:e1760.
[4] Becker N, Schön S, Klein A, Ferstl I, Kizgin A, Tannich E, Kuhn C, Pluskota B, Jöst A. First mass development of Aedes albopictus (Diptera: Culicidae)-its surveillance and control in Germany. Parasitology research 2017; 116(3):847-858.

[5] Kampen H, Schäfer M, Scheuch D, Werner D. Further specimens of the Asian tiger mosquito Aedes albopictus (Diptera, Culicidae) trapped in southwest Germany. Parasitology research 09 2012; 112(2):905-907.

[6] Zur Situation bei wichtigen Infektionskrankheiten Reiseassoziierte Krankheiten 2018. Epidemiologisches Bulletin - Robert Koch-Institut 2019; (1):513-524.

[7] Rodrigues HS. Optimal Control and Numerical Optimization Applied to Epidemiological Models. PhD Thesis, University Aveiro, Portugal 2012.

[8] Rodrigues HS, Monteiro MTT, Torres DF. Bioeconomic perspectives to an optimal control dengue model. International Journal of Computer Mathematics 2013; 90(10):2126-2136.

[9] Fischer A, Chudej K, Pesch HJ. Optimal vaccination and control strategies against dengue. Mathematical Methods in the Applied Sciences 2019; 42(10):34963507.

[10] Castillo-Chavez C, Hethcote HW, Andreasen V, Levin SA, Liu WM. Epidemiological models with age structure, proportionate mixing, and cross-immunity. Journal of Mathematical Biology 1989; 27(3):233-258.

[11] Feng Z, Velasco-Hernández JX. Competitive exclusion 

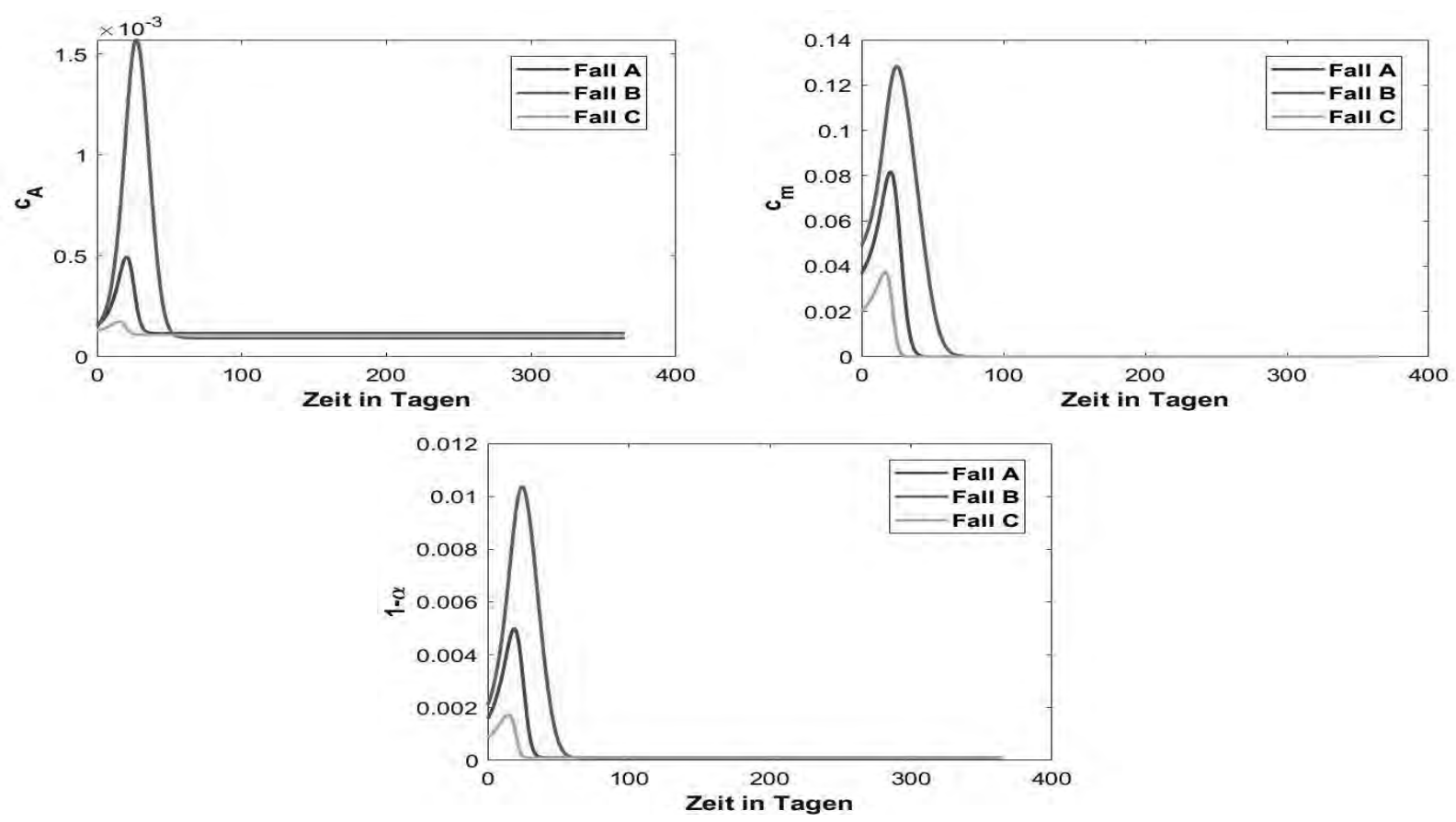

Abbildung 5: Einsatz von Larvizid (links), Adultizid (rechts) und mechanischer Kontrolle (unten)

in a vector-host model for the dengue fever. Journal of Mathematical Biology 1997; 35(5):523-544.

[12] Esteva L, Vargas C. Coexistence of different serotypes of dengue virus. Journal of Mathematical Biology 2003; 46(1):31-47.

[13] Chudej K, Fischer A. Optimal Vaccination Strategies for a new Dengue Model with two Serotypes. IFACPapersOnLine 2018; 51(2):13-18.

[14] Mitkowski W. Dynamical properties of Metzler systems. Bulletin of the Polish Academy of Sciences: Technical Sciences 2008; 56(4):309-312.

[15] Abate A, Tiwari A, Sastry S. Box invariance in biologically-inspired dynamical systems. Automatica 2009; 45(7):1601-1610.

[16] Van den Driessche P, Watmough J. Reproduction numbers and sub-threshold endemic equilibria for compartmental models of disease transmission. Mathematical Biosciences 2002; 180(1-2):29-48.

[17] Heffernan J, Smith R, Wahl L. Perspectives on the basic reproductive ratio. Journal of the Royal Society 10 2005; 2:281-93.

[18] van den Driessche P, Watmough J. Further Notes on the Basic Reproduction Number. Springer: Berlin, 2008; 159-178.

[19] Van den Driessche P. Reproduction numbers of infectious disease models. Infectious Disease Modelling 2017; 2(3):288-303.
[20] Albrecht G, Fischer A, Chudej K. Analyse, Simulation und Optimale Steuerung eines mathematischen Dengue-Fieber Modells mit Impfung. Tagungsband Workshop Heilbronn 2018 ASIM/GI Fachgruppen, ARGESIM Report, vol. 54. ARGESIM Verlag: Wien, Österreich, 2018; 223-229.

[21] Mordecai EA, Cohen JM, Evans MV, Gudapati P, Johnson LR, Lippi CA, Miazgowicz K, Murdock CC, Rohr JR, Ryan SJ, et al.. Detecting the impact of temperature on transmission of Zika, dengue, and chikungunya using mechanistic models. PLoS Neglected Tropical Diseases 2017; 11(4):e0005 568.

[22] Herath M, Albrecht G, Chudej K. Ein asymmetrisches zwei Serotyp Dengue Fieber Modell mit Kontrollmaßnahmen. Simulation in Umwelt- und Geowissenschaften: Workshop 2020, ASIM Mitteilung, vol. 173. Shaker Verlag: Düren, 2020; 191-202.

[23] Fourer R, Gay D, Kernighan B. AMPL: A Modeling Language for Mathematical Programming, vol. 36. Duxbury Press: Pacific Grove, 2002.

[24] Wächter A, Biegler L. On the implementation of an interior-point filter line-search algorithm for large-scale nonlinear programming. Mathematical programming 03 2006; 106:25-57. 